\title{
Maximum-Likelihood Channel Estimation in Block Fading Amplify-and-Forward Relaying Networks
}

\author{
Nico Aerts, Iancu Avram and Marc Moeneclaey \\ Department of Telecommunications \\ and Information Processing \\ Ghent University \\ 9000 Gent, Belgium \\ Email: firstname.lastname@telin.ugent.be
}

\begin{abstract}
Several diversity techniques have been proposed to counteract the effect of fading on the error performance of wireless networks. A recent and promising technique, which achieves spatial diversity without increased hardware demands, is cooperative communication, involving other terminals in the network that relay the information broadcasted by the source terminal to the destination terminal. In literature several cooperative protocols have been studied under the simplifying assumption that all channel state information is available at the destination. In this paper, we use the space-alternating generalized expectation-maximization (SAGE) algorithm to perform codeaided iterative channel estimation from the broadcasted signals in an Amplify-and-Forward protocol, and investigate the resulting error performance.
\end{abstract}

Index Terms-Cooperative Communication, Amplify-andForward, SAGE Algorithm, Maximum-Likelihood

\section{INTRODUCTION}

In wireless networks with multipath propagation, the reliability of the communication system is determined by the probability that the channel between the source and the destination is in a deep fade. The detrimental effect of fading can be counteracted by creating multiple independent channels using spatial, temporal or frequency diversity techniques [1], in which case the reliability of the system is determined by the probability that all channels are simultaneously experiencing deep fading. A promising and relatively new method to obtain spatial diversity in a wireless network is cooperative communication [2], [3]. This involves utilizing other terminals for relaying to the destination information that is related to the message sent by the source. In literature several cooperative protocols have been proposed, such as Amplify-and-Forward (AF), Decode-and-Forward [4], Quantize-and-Forward [5], [6] and coded cooperation [7]. In this contribution we will concentrate on the AF protocol where the relay simply amplifies and retransmits the data signal it receives from the source.

In reality channel state information is not available and needs to be estimated. Therefore the source sends pilot symbols in addition to the data symbols; the received signals corresponding to the pilot symbols are used to obtain the required estimates. This type of estimation is called pilot-aided estimation and is applied to the AF protocol in e.g. [8], [9] and [10]. Unfortunately, the drawback of this estimation strategy is that a large number of pilot symbols are needed, thus deteriorating power and bandwidth efficiency. Instead, in this paper we will use only a few pilot symbols to derive initial estimates, and improve these estimates iteratively by means of the space-alternating generalized expectation-maximization (SAGE) algorithm [11], [12] which exploits the presence of the unknown (coded) data symbols.

This contribution is organized as follows. The model of the AF system is described in Section II. Section III presents the derivation of the pilot-aided and of the SAGE-based channel estimation algorithms. Numerical results regarding the estimator performance and the frame error rate are given in Section IV. Finally, conclusions are drawn in section V.

\section{NOTATIONS}

All vectors are row vectors and boldface; the Hermitian transpose, statistical expectation, estimate and real and imaginary part of the row vector $\boldsymbol{x}$ are denoted by $\boldsymbol{x}^{H}, \mathbb{E}[\boldsymbol{x}], \hat{\boldsymbol{x}}$, $\Re\{\boldsymbol{x}\}$ and $\Im\{\boldsymbol{x}\}$ respectively; $[\boldsymbol{x}, \boldsymbol{y}]$ denotes the concatenation of $\boldsymbol{x}$ and $\boldsymbol{y} ;(\mathbf{X})_{k, m}$ is the $(k, m)$ th element of the matrix $\mathrm{X}$; the cardinality of the set $\mathbb{X}$ is denoted as $|\mathbb{X}|$; the complex conjugate of a scalar $x$ is denoted $x^{*}$.

\section{System Description}

In a cooperative system the time frame allocated to send information from a source terminal to its destination terminal is divided into $\mathrm{L}+1$ time slots. The source utilizes only the first slot to broadcasts the information. The remaining $L$ time slots are used by $\mathrm{L}$ other terminals to relay this information to the destination in nonoverlapping slots. In this contribution we restrict ourselves to systems with only one relay as the extension to multiple relays is straightforward. The network now contains 3 terminals as depicted in Fig. 1.

During the first time slot, the source $S$ broadcasts a vector $\boldsymbol{c}=\left(\boldsymbol{c}_{S, p}, \boldsymbol{c}_{d}\right)$ of $K$ symbols, consisting of $K_{S, p}$ pilot symbols (denoted $\boldsymbol{c}_{S, p}$ ) and $K_{d}$ data symbols (denoted $\boldsymbol{c}_{\boldsymbol{d}}$ ), with $K=K_{S, p}+K_{d}$. The pilot symbols are included to assist the estimation of the channel parameters (see section III). The data symbols $c_{d}$ are obtained by first encoding (at rate $k / n)$ a vector of information bits $\boldsymbol{b}$, and subsequently 


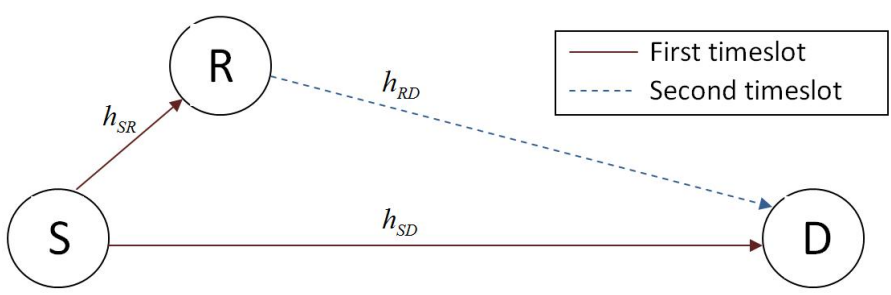

Fig. 1. Network containing a source S, a relay R and a destination D.

mapping the encoder output onto a constellation $\mathbb{S}$. The energy per pilot symbol and the energy per data symbol take the same value $E_{s}$. The average transmitted energy per symbol $E_{s}$ and the symbol rate $R_{s}$ are related to the transmitted energy per information bit $E_{b}$ and the information bitrate $R_{b}$ by

$$
\begin{aligned}
E_{s} & =\frac{K_{d}}{K_{S, p}+K_{d}} \frac{k}{n} \log _{2}(|\mathbb{S}|) E_{b}, \\
R_{s} & =\frac{K_{S, p}+K_{d}}{K_{d}} \frac{n}{k} \frac{R_{b}}{\log _{2}(|\mathbb{S}|)} .
\end{aligned}
$$

For given $E_{b}$ and $R_{b}$, the inclusion of pilot symbols reduces $E_{s}$ (loss of power efficiency for detection) and increases $R_{s}$ (loss of spectral efficiency).

We assume slow independent frequency flat Rayleigh fading channels with additive white Gaussian noise and represent them by means of channel coefficients $h_{i}$ and noise vectors $\boldsymbol{w}_{i}(i \in\{S D, S R, R D\})$. Here $h_{i}$ and the elements of the noise vectors $\boldsymbol{w}_{i}$ are independent zero-mean circular symmetric complex Gaussian (ZMCSCG) random variables with variances $H_{i}$ and $N_{0, i}$ respectively. The indices SR, SD and $\mathrm{RD}$ refer to the source-relay (S-R), source-destination (S-D) and relay-destination (R-D) channels, respectively. During the first time slot, the signal received by the relay $\mathrm{R}$ is given by $\boldsymbol{r}_{S R}=\left(\boldsymbol{r}_{S R, p}, \boldsymbol{r}_{S R, d}\right)$, with

$$
\begin{aligned}
& \boldsymbol{r}_{S R, p}=h_{S R} \boldsymbol{c}_{S, p}+\boldsymbol{w}_{S R, p}, \\
& \boldsymbol{r}_{S R, d}=h_{S R} \boldsymbol{c}_{d}+\boldsymbol{w}_{S R, d},
\end{aligned}
$$

and $\boldsymbol{w}_{S R}=\left(\boldsymbol{w}_{S R, p}, \boldsymbol{w}_{S R, d}\right)$. Similarly the signal $\boldsymbol{r}_{S D}$ received during the first time slot at the destination $\mathrm{D}$ is $\boldsymbol{r}_{S D}=$ $\left(\boldsymbol{r}_{S D, p}, \boldsymbol{r}_{S D, d}\right)$, with

$$
\begin{aligned}
& \boldsymbol{r}_{S D, p}=h_{S D} \boldsymbol{c}_{S, p}+\boldsymbol{w}_{S D, p}, \\
& \boldsymbol{r}_{S D, d}=h_{S D} \boldsymbol{c}_{d}+\boldsymbol{w}_{S D, d},
\end{aligned}
$$

and $\boldsymbol{w}_{S D}=\left(\boldsymbol{w}_{S D, p}, \boldsymbol{w}_{S D, d}\right)$. The relay makes use of $\boldsymbol{r}_{S R, p}$ to make a pilot-aided estimate $\hat{h}_{S R}$ of $h_{S R}$ (see section III). During the second time slot, the relay transmits $K_{R, p}$ pilot symbols $\boldsymbol{c}_{R, p}$ (with average energy per symbol $E_{r}$ ), $K_{e}$ times the estimate $\hat{h}_{S R}$, and finally the signal $\boldsymbol{r}_{S R, d}$ received from the source. We select $K_{R, p}+K_{e}=K_{S, p}$, such that both slots have the same symbol rate $R_{s}$ and the same duration $K / R_{s}$. Hence, the signal received by the destination during the second time slot is $\boldsymbol{r}_{R D}=\left(\boldsymbol{r}_{R D, p}, \boldsymbol{r}_{R D, e}, \boldsymbol{r}_{R D, d}\right)$, with

$$
\begin{aligned}
\boldsymbol{r}_{R D, p} & =\alpha h_{R D} \boldsymbol{c}_{R, p}+\boldsymbol{w}_{R D, p}, \\
\boldsymbol{r}_{R D, e} & =\beta \sqrt{E_{r}} h_{R D} \hat{h}_{S R} 1_{K_{e}}+\boldsymbol{w}_{R D, e}, \\
\boldsymbol{r}_{R D, d} & =\gamma h_{R D} \boldsymbol{r}_{S R, d}+\boldsymbol{w}_{R D, d} \\
& =\gamma h_{R D} h_{S R} \boldsymbol{c}_{d}+\gamma h_{R D} \boldsymbol{w}_{S R, d}+\boldsymbol{w}_{R D, d},
\end{aligned}
$$

and where $1_{M}$ denotes a row vector consisting of $\mathrm{M}$ ones, while $\alpha, \beta$ and $\gamma$ are gain factors of the relay. Imposing the average energy transmitted by the relay to be $K E_{r}, \gamma$ is expressed as

$$
\gamma=\sqrt{\frac{K E_{r}-\alpha^{2} K_{R, p} E_{r}-\beta^{2} K_{e} E_{r}\left(H_{S R}+\frac{N_{0, S R}}{K_{S, p} E_{s}}\right)}{H_{S R} K_{d} E_{s}+K_{d} N_{0, S R}}}
$$

In order to obtain the information bit decisions $\hat{b}$, the signals $\boldsymbol{r}_{S D, d}$ and $\boldsymbol{r}_{R D, d}$ should be properly combined and applied to the decoder. When perfect channel state information is available at the destination, the sufficient statistic $\boldsymbol{\eta}$ to be applied to the decoder is given by

$$
\boldsymbol{\eta}=\frac{h_{S D, d}^{*} \boldsymbol{r}_{S D, d}}{N_{0, S D}}+\frac{\gamma h_{S R}^{*} h_{R D}^{*} \boldsymbol{r}_{R D}}{N_{0, R D}+\gamma^{2}\left|h_{R D}\right|^{2} N_{0, S R}} .
$$

Note that (4) corresponds to maximum-ratio combining [13]. When convolutional or trellis encoding [14] is used, decoding can be accomplished by means of the Viterbi algorithm (ML sequence detection) [15] or the BCJR algorithm (MAP bit detection) [16].

\section{Channel Estimation}

We assume the noise variances $N_{0, i}(i \in\{S D, S R, R D\})$ to be known as these are long-term properties. Based on $\boldsymbol{r}_{S R, p}$, the relay makes a pilot-aided ML estimate of $h_{S R}$, according to

$$
\hat{h}_{S R}=\arg \max _{h_{S R}} \ln p\left(\boldsymbol{r}_{S R, p} \mid h_{S R}\right)=\frac{\boldsymbol{r}_{S R, p} \boldsymbol{c}_{S, p}^{H}}{\left|\boldsymbol{c}_{S, p}\right|^{2}} .
$$

It is easily verified that $\hat{h}_{S R}=h_{S R}+n_{e}$, with $\mathbb{E}\left[\left|n_{e}\right|^{2}\right]$ $=N_{0, S R} /\left|\boldsymbol{c}_{S, p}\right|^{2}$. Substituting (5) in (2), we observe that $\boldsymbol{r}_{S D}$ and $\boldsymbol{r}_{R D}$ depend only on $h_{S D}, h_{S R D}=h_{S R} h_{R D}$ and $h_{R D}$, which are the parameters needed to calculate the sufficient statistic (4). Note that $h_{S R D}$ is the channel gain of the compound S-R-D channel.

Our parameter estimation strategy at the destination consists of first determining an initial estimate of the unknown parameter vector $\left(h_{S D}, h_{S R D}, h_{R D}\right)$ based on the observations $\left(\boldsymbol{r}_{S D, p}, \boldsymbol{r}_{R D, p}, \boldsymbol{r}_{R D, e}\right)$. Further, this initial estimate is iteratively refined by means of the SAGE algorithm, that takes the entire observation $\boldsymbol{r}_{D}=\left(\boldsymbol{r}_{S D}, \boldsymbol{r}_{R D}\right)$ at the destination into account. The detection of $\boldsymbol{c}_{d}$ is based upon the sufficient statistic (4), but with $h_{S D}, h_{S R D}$ and $h_{R D}$ replaced by their estimates. 


\section{A. Initial Estimates}

From the properties of $\hat{h}_{S R}$, it follows that $\boldsymbol{r}_{R D, e}=$ $\beta \sqrt{E_{r}} h_{S R D} 1_{K_{e}}+\beta \sqrt{E_{r}} h_{R D} n_{e} 1_{K_{e}}+\boldsymbol{w}_{R D, e}$. As the covariance matrix of the noise term $\beta \sqrt{E_{r}} h_{R D} n_{e} 1_{K_{e}}+\boldsymbol{w}_{R D, e}$ in $\boldsymbol{r}_{R D, e}$ is not diagonal, we consider a linear transformation $\boldsymbol{r}_{R D, e} \mathrm{~V}$ that yields uncorrelated noise components, where $\mathrm{V}$ is a non-singular $K_{e} \mathrm{x} K_{e}$ matrix. This is achieved by taking the first column of $\mathrm{V}$ proportional to $1_{K_{e}}^{T}$, and taking vectors spanning the subspace that is orthogonal to $1_{K_{e}}^{T}$ as the remaining $K_{e}-1$ columns of $\mathrm{V}$. The first element of $\boldsymbol{r}_{R D, e} \mathrm{~V}$, denoted $z_{R D, e}$, is a sufficient statistic, as it is the only element of $\boldsymbol{r}_{R D, e} \mathrm{~V}$ that depends on $\left(h_{S R D}, h_{R D}\right)$. We obtain

$$
z_{R D, e}=\frac{\boldsymbol{r}_{R D, e} 1_{K_{e}}^{T}}{K_{e}}=\beta \sqrt{E_{r}} h_{S R D}+\beta \sqrt{E_{r}} h_{R D} n_{e}+n_{R D, e}
$$

with $\mathbb{E}\left[\left|n_{R D, e}\right|^{2}\right]=N_{0, R D} / K_{e}$. The initial estimates of $h_{S D}$ and $h_{R D}$ are pilot-aided ML estimates based on $\boldsymbol{r}_{S D, p}$ and $\boldsymbol{r}_{R D, p}$ :

$$
\begin{gathered}
\hat{h}_{S D}^{(0)}=\arg \max _{h_{S D}} \ln p\left(\boldsymbol{r}_{S D, p} \mid h_{S D}\right)=\frac{\boldsymbol{r}_{S D, p} \boldsymbol{c}_{S, p}^{H}}{\left|\boldsymbol{c}_{S, p}\right|^{2}}, \\
\hat{h}_{R D}^{(0)}=\arg \max _{h_{R D}} \ln p\left(\boldsymbol{r}_{R D, p} \mid h_{R D}\right)=\frac{\boldsymbol{r}_{R D, p} \boldsymbol{c}_{R, p}^{H}}{\alpha\left|\boldsymbol{c}_{R, p}\right|^{2}},
\end{gathered}
$$

whereas the initial ML estimate of $h_{S R D}$ is based on $z_{R D, e}$ :

$$
\hat{h}_{S R D}^{(0)}=\arg \max _{h_{S R D}} \ln p\left(z_{R D, e} \mid h_{S R D}\right)=\frac{z_{R D, e}}{\beta \sqrt{E_{r}}} .
$$

\section{B. SAGE-algorithm}

When also exploiting $\boldsymbol{r}_{S D, d}$ and $\boldsymbol{r}_{R D, d}$ for channel estimation, one has to deal with the nuisance parameter $\boldsymbol{c}_{d}$. This can be taken care of by using the expectation-maximization (EM) algorithm [17]. When estimating from an observation $r$ a vector parameter $\boldsymbol{\theta}$ in the presence of a nuisance parameter $\boldsymbol{c}$, the EM-algorithm produces a sequence of estimates $\hat{\boldsymbol{\theta}}^{(i)}$, $i=1,2, \ldots$, according to

$$
\hat{\boldsymbol{\theta}}^{(i)}=\arg \max _{\boldsymbol{\theta}} \mathbb{E}\left[\ln p(\boldsymbol{r} \mid \boldsymbol{\theta}, \boldsymbol{c}) \mid \boldsymbol{r}, \hat{\boldsymbol{\theta}}^{(i-1)}\right],
$$

where the conditional expectation is with respect to the nuisance parameter $\boldsymbol{c}$. An estimate $\hat{\boldsymbol{\theta}}^{(0)}$ is required to initialize the EM iterations.

As the likelihood function at the destination is the product of $p\left(\boldsymbol{r}_{S D} \mid h_{S D}, \boldsymbol{c}\right)$ and $p\left(\boldsymbol{r}_{R D} \mid h_{S R D}, h_{R D}, \boldsymbol{c}\right)$, the EM algorithm yields, with $\boldsymbol{\theta}=\left(h_{S D}, h_{S R D}, h_{R D}\right)$ and $\boldsymbol{r}=\left(\boldsymbol{r}_{S D}, \boldsymbol{r}_{R D}\right)$,

$$
\begin{gathered}
\hat{h}_{S D}^{(i)}=\arg \max _{h_{S D}} \mathbb{E}\left[\ln p\left(\boldsymbol{r}_{S D} \mid h_{S D}, \boldsymbol{c}_{d}\right) \mid \boldsymbol{r}, \hat{\boldsymbol{\theta}}^{(i-1)}\right], \\
=\frac{\boldsymbol{r}_{S D, p} \boldsymbol{c}_{S, p}^{H}+\boldsymbol{r}_{S D, d}\left(\boldsymbol{m}_{1, d}^{(i-1)}\right)^{H}}{\left|\boldsymbol{c}_{S, p}\right|^{2}+m_{2, d}^{(i-1)}}, \\
\left(\hat{h}_{S R D}^{(i)}, \hat{h}_{R D}^{(i)}\right)= \\
\arg \max _{h_{S R D}, h_{R D}} \mathbb{E}\left[\ln p\left(\boldsymbol{r}_{R D} \mid h_{S R D}, h_{R D}, \boldsymbol{c}_{d}\right) \mid \boldsymbol{r}, \hat{\boldsymbol{\theta}}^{(i-1)}\right]
\end{gathered}
$$

where $\boldsymbol{m}_{1, d}^{(i)}$ and $m_{2, d}^{(i)}$ are the a posteriori expectations of $\boldsymbol{c}_{d}$ and $\left|\boldsymbol{c}_{d}\right|^{2}$ :

$$
\begin{array}{r}
\boldsymbol{m}_{1, d}^{(i)}=\mathbb{E}\left[\boldsymbol{c}_{d} \mid \boldsymbol{r}_{d}, \hat{\boldsymbol{\theta}}^{(i-1)}\right], \\
m_{2, d}^{(i)}=\mathbb{E}\left[\left|\boldsymbol{c}_{d}\right|^{2} \mid \boldsymbol{r}_{d}, \hat{\boldsymbol{\theta}}^{(i-1)}\right] .
\end{array}
$$

These a posteriori expectations can be computed from the marginal a posteriori probabilities of the elements of $\boldsymbol{c}_{d}$, which result from the decoder. In order to avoid the complexity of the two-dimensional maximization associated with (12), we will use the SAGE algorithm [11] rather than the EM algorithm. The SAGE algoritm replaces the two-dimesional maximization in (12) by two one-dimensional maximizations : during the $\mathrm{i}^{\text {th }}$ iteration, we first maximize over $h_{S R D}$ with $h_{R D}=\hat{h}_{R D}^{(i-1)}$, then we maximize over $h_{R D}$ with $h_{S R D}=\hat{h}_{S R D}^{(i)}$. For the estimation of $h_{S R D}$ this yields

$$
\hat{h}_{S R D}^{(i)}=\frac{\frac{\beta \sqrt{E_{r}} z_{R D, e}}{\operatorname{Var}_{R D, e}^{(i-1)}}+\frac{\gamma \boldsymbol{r}_{R D, d}\left(\boldsymbol{m}_{1, d}^{(i-1)}\right)^{H}}{\operatorname{Var}_{R D, d}^{(i-1)}}}{\frac{\beta^{2} E_{r}}{\operatorname{Var}_{R D, e}^{(i-1)}}+\frac{\gamma^{2} m_{2, d}^{(i-1)}}{\operatorname{Var}_{R D, d}^{(i-1)}}},
$$

with

$$
\begin{aligned}
\operatorname{Var}_{R D, e}^{(i-1)} & =\frac{N_{0, R D}}{K_{e}}+\beta^{2} E_{r}\left|\hat{h}_{R D}^{(i)}\right|^{2} \frac{N_{0, S R}}{\left|\boldsymbol{c}_{S, p}\right|^{2}} \\
\operatorname{Var}_{R D, d}^{(i-1)} & =N_{0, R D}+\gamma^{2}\left|\hat{h}_{R D}^{(i)}\right|^{2} N_{0, S R}
\end{aligned}
$$

The maximization in (12) over $h_{R D}$ (with $h_{S R D}=\hat{h}_{S R D}^{(i)}$ ) does not give rise to a closed-form expression for $\hat{h}_{R D}^{(i)}$, because both the mean (see the term $\alpha h_{R D} c_{R, p}$ in (1)) and the covariance matrix (see the terms $\gamma h_{R D} \boldsymbol{w}_{S R, d}$ and $\beta \sqrt{E_{r}} h_{R D} n_{e}$ in (3) and (6), respectively) of $\boldsymbol{r}_{R D}$ are functions of $h_{R D}$. This problem can be circumvented by replacing this covariance matrix by its value corresponding to $h_{R D}=\hat{h}_{R D}^{(i-1)}$. Because of this substitution, only the mean of $\boldsymbol{r}_{R D}$ depends (linearly) on $h_{R D}$, so that

$$
\hat{h}_{R D}^{(i)}=\frac{\boldsymbol{r}_{R D, p} \boldsymbol{c}_{R, p}^{H}}{\alpha\left|\boldsymbol{c}_{R, p}\right|^{2}} .
$$

Observe the estimate of $h_{R D}$ to remain unchanged during the iterations, as (16) equals the initial estimate (8) of $h_{R D}$. In this case, the SAGE algorithm reduces to a maximization over $h_{S R D}$ only.

\section{Simulation Results}

In this section, we study the performance of the $\mathrm{AF}$ communication system. By means of computer simulations we determine the mean-square estimation error (MSEE) of the channel parameter estimates and the associated Frame Error Rate (FER) of the detector, averaged over many realizations of $h_{S D}, h_{S R}$ and $h_{R D}$. Unless otherwise specified, a rate- $\frac{1}{2}(15,17)$ convolutioal code is used, the coded bits are Gray mapped onto 4-PSK symbols ${ }^{1}$ and a fixed number of $K_{d}=260$ data symbols are transmitted. As

\footnotetext{
${ }^{1}$ Note that $m_{2, d}^{(i)}=K_{d} E_{s}$ for M-PSK.
} 
it is likely for the terminals in the network to have nearly identical front-ends, we assume the noise variances to be equal. We choose the noise variances to be normalized, i.e. $N_{S R}=N_{R D}=N_{S D}=1$ and the power constraints at the source and the relay to be identical, i.e. $E_{r}=E_{s}$. The channel gain variances are determined by $2 H_{S R}=2 H_{R D}=H_{S D}=1$, so that the S-R channel and the R-D channel have the same SNR, which is $3 \mathrm{~dB}$ higher than the SNR on the S-D channel. Unless otherwise specified, we will always use 3 SAGE iterations.

The parameters $\alpha, \beta$ and $K_{e}$ determine how much energy of the frame sent by the relay is devoted to the transmission of pilot symbols, of the estimate of $h_{S R}$, and of the data symbols. Obviously, a compromise exists between guaranteeing good initial estimates at the destination while maintaining a high enough data symbol energy for detection. We have selected $\alpha=\beta=1$ and $K_{e}=\left\lfloor K_{p} / 2\right\rfloor$, from which the values for $\gamma$ and $K_{R, p}=K_{S, p}-K_{e}$ follow. Fig. 2 depicts the

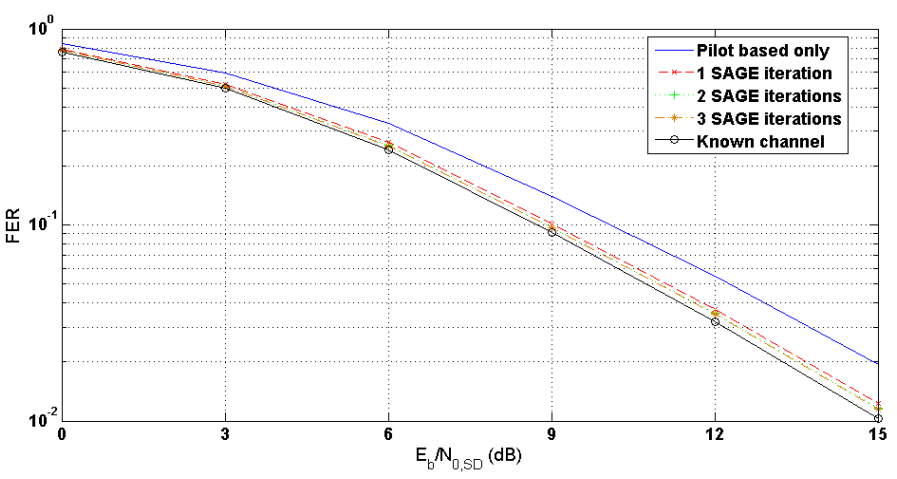

Fig. 2. FER with $K_{p}=5, K_{e}=2, \alpha=1$ and $\beta=1$

FER in function of the SNR on the S-D channel. Note that only a few SAGE iterations are needed for the algorithm to converge. In the high-SNR region the FER performance resulting from the SAGE algorithm (3 iterations) is degraded by only $0.34 \mathrm{~dB}$ as compared to the FER of the system that knows the channel parameters, whereas a degradation of $1.47 \mathrm{~dB}$ is observed when the channel estimation uses pilot symbols only. These degradations include the power efficiency loss due the presence of pilot symbols, which amounts to $10 \log _{10}\left(\frac{265}{260}\right)=0.08 \mathrm{~dB}$. Hence, the SAGE algorithm achieves a gain in the order of $1.1 \mathrm{~dB}$ over pilot based estimates.

In the extreme case where only $K_{S, p}=2$ pilot symbols are used, and with $K_{e}=1$, the following degradations at high SNR are observed: $3.65 \mathrm{~dB}$ (pilot symbols only) and 1.00 dB (3 SAGE iterations). Here the SAGE algorithm yields a gain in the order of $2.6 \mathrm{~dB}$ over pilot based estimates, but the resulting frame error rate is larger than in the case where $\left(K_{S, p}, K_{e}\right)=(5,2)$.

The Cramer-Rao bound (CRB) [18] and the modified CRB [19] are fundamental lowerbounds on the MSEE of unbiased estimates, and therefor, a useful benchmark for the actual MSEE. Although the MCRB is a looser bound than the CRB, they have the same high-SNR limit [20]. As a result, the obtained closed form expressions of the MCRBs capture the high-SNR behavior of the true CRBs. For the derivation of these bounds we refer to [21] where a similar communication system is considered. The initial estimates from $(5,7-9)$ are unbiased and their MSEE coincides with the CRB that corresponds to the observation of $\left(\boldsymbol{r}_{S D, p}, \boldsymbol{r}_{R D, p}\right.$ and $\left.z_{R D, e}\right)$. Hence the initial estimates have the smallest MSEE possible. In most cases however, the CRB based on the entire observation $\left(\boldsymbol{r}_{S D}\right.$, $\boldsymbol{r}_{R D}$ ) is not achievable, because of the presence of the nuisance parameters $\boldsymbol{c}_{d}$. Fig. 3 depicts the MSEE of the estimate of

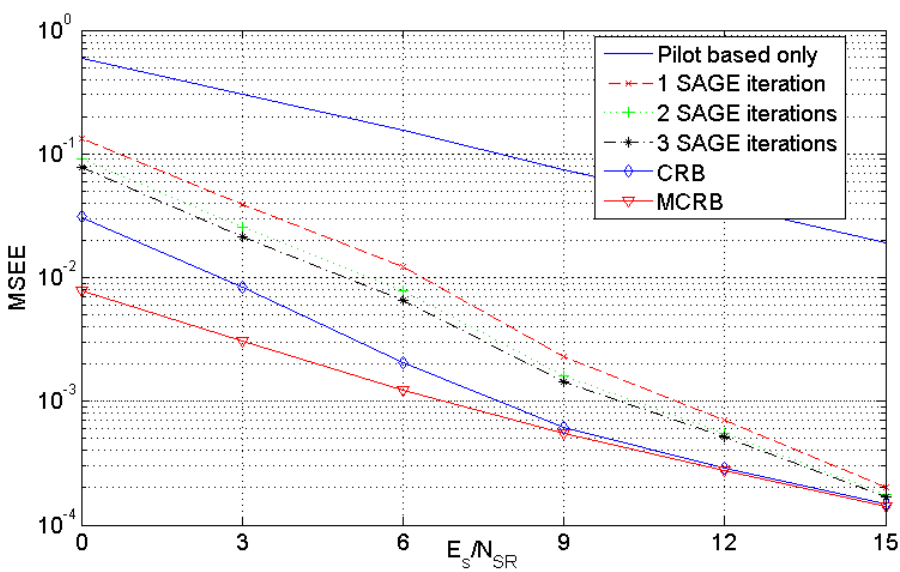

Fig. 3. MSEE related to the estimate of $h_{S R D}$, with $K_{p}=5, K_{e}=2$, $\alpha=1$ and $\beta=1$

$h_{S R D}$ along with the corresponding CRB and the MCRB based on the entire observation $\left(\boldsymbol{r}_{S D}, \boldsymbol{r}_{R D}\right)$. The displayed MSEE, CRB and MCRB represent an average over many realizations of $\left(h_{S D}, h_{S R}, h_{R D}\right)$. Observe the performance of the SAGE algorithm to converge to these lowerbounds in the high SNRregion. For given $h_{R D}$ the MSEE of the initial estimate of $h_{S R D}$ equals

$$
\mathbb{E}\left[\left|h_{S R D}-\hat{h}_{S R D}^{(0)}\right|^{2}\right]=\frac{\left|h_{R D}\right|^{2} N_{0, S R}}{\left|\boldsymbol{c}_{S, p}\right|^{2}}+\frac{N_{0, R D}}{K_{e} \beta^{2} E_{r}} .
$$

and due to the small values of $K_{e}$ and $K_{S, p}$, rather high values for this initial MSEE are obtained, as compared to the MSEE of the iterative estimates. The MCRB turns out to be obtained by replacing in (15) the soft decisions $\boldsymbol{m}_{1, d}^{(i-1)}$ by the true data symbols $\boldsymbol{c}_{\boldsymbol{d}}$ and by replacing the estimate of $h_{R D}$ by its true value. This results in

$$
\left(\frac{\beta^{2} E_{r}}{\frac{N_{0, R D}}{K_{e}}+\beta^{2} E_{r}\left|h_{R D}\right|^{2} \frac{N_{0, S R}}{\left|\boldsymbol{c}_{S, p}\right|^{2}}}+\frac{\gamma^{2}\left|\boldsymbol{c}_{d}\right|^{2}}{N_{0, R D}+\gamma^{2}\left|h_{R D}\right|^{2} N_{0, S R}}\right)^{-1}
$$

Fig. 4 shows the performance results (averaged over many realizations of $\left.\left(h_{S D}, h_{S R}, h_{R D}\right)\right)$ corresponding to the estimation of $h_{S D}$. As in Fig. 3, the MSEE improves during the iterations, and for large SNR is close to the CRB and 
the MCRB related to the estimation of $h_{S D}$, based on the entire observation $\left(\boldsymbol{r}_{S D}, \boldsymbol{r}_{R D}\right)$. According to (16), the initial

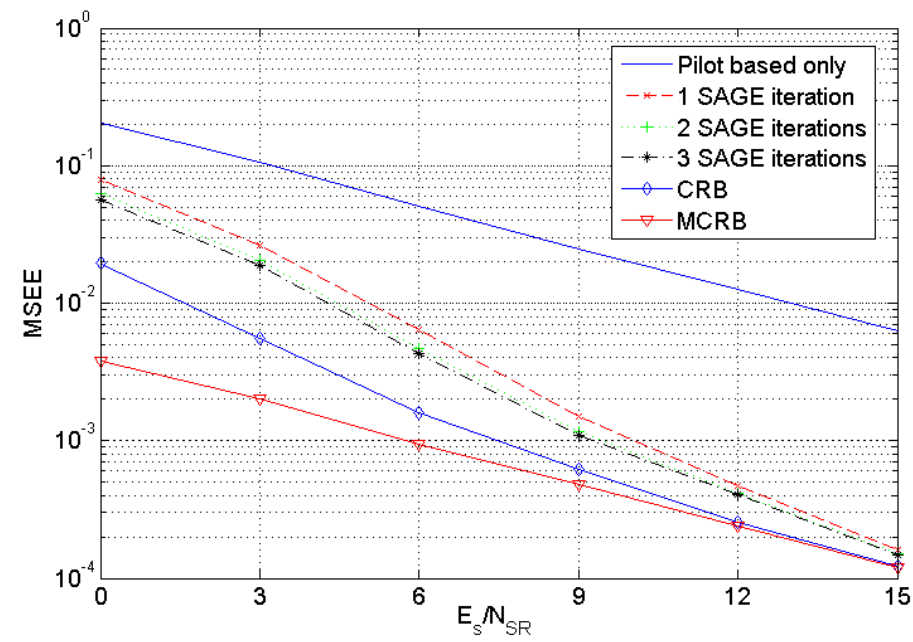

Fig. 4. MSEE related to the estimate of $h_{S D}$, with $K_{p}=5, K_{e}=2$, $\alpha=1$ and $\beta=1$

estimate of $h_{S R}$ is not further refined during the iterations. The corresponding MSEE equals $N_{0, R D} /\left(\alpha^{2} E_{r} K_{R, p}\right)$, which becomes rather large (as compared to the MSEE related to the iterative estimation of $h_{S D}$ and $h_{S R D}$ ) for small $K_{R, p}$.

Considering (4), it is important that the estimates of $h_{S D}$ and $h_{S R D}$ improve during the iterations, because these estimates are needed to compensate the rotation of the data symbols on the S-D and S-R-D channels. The estimate of $h_{R D}$ does not change during the iterations (see (16)). The limited accuracy (caused by a small value of $K_{R, p}$ ) of the estimate of $h_{R D}$ is not very critical, as it only affects the weight (but not the phase) of the second term in (4).

\section{CONCLUSIONS AND REMARKS}

In this contribution we have investigated an iterative estimation algorithm for the cooperative Amplify-andForward protocol and studied the overall performance of the system in terms of MSEE and FER. We showed that this iterative approach yields a substantial gain over pilot based estimates and that only a few pilot-symbols and iterations are needed to achieve a FER performance close to the one of the system that knows the channels.

We have not attempted to optimize the parameters $\alpha, \beta$ and $K_{e}$ at the relay, as a function of the SNR's on the S-D, S-R and R-D channels, but simply selected $\alpha=1, \beta=1$ and $K_{e}=\left\lfloor K_{p} / 2\right\rfloor$. Taking into account that the resulting FER is only about $0.34 \mathrm{~dB}$ worse than the FER that corresponds to known channel parameters, the potential gain from such optimization is quite small.

The channel estimates presented in this paper have been for block fading channels. Future work will involve the derivation and analysis of the channel estimates in the (more challenging) case of slow time-selective fading.

\section{ACKNOWLEDGMENT}

The authors wish to acknowledge the activity of the Network of Excellence in Wireless COMmunications NEW$\mathrm{COM}++$ of the European Commission (contract n. 216715) that motivated this work.

\section{REFERENCES}

[1] J. G. Proakis, Digital Communications. New York: McGraw-Hill, 1995. [2] J. N. Laneman, "Cooperative Diversity in Wireless networks: Algorithms and Architectures," Ph.D. dissertation, Massachusetts Institute of Technology, Massachusetts, USA, 2002

[3] A. Sendonaris, E. Erkip and B. Aazhang, "User Cooperation DiversityPart I: System Description," IEEE Trans. Commun., vol. 51, no. 11, pp. 1927-1938, Nov. 2003

[4] T. Cover and A. E. Gamal, "Capacity Theorems for the Relay Channel," IEEE Trans. Inform. Theory, vol. 25, no. 5, pp. 572-584, Sep. 1979

[5] M. Souryal and H. You, "Quantize-and-Forward Relaying with Mary Phase Shift Keying,' Wireless Communication and Networking Conference, 2008, pp. 42-47

[6] A. Sendonaris, E. Erkip and B. Aazhang, "Practical Quantize-andForward Schemes for the Frequency Division Relay Channel,' EURASIP Journal on Wireless Communications and Networking, vol. 2007, Article ID 20258, 11 pages

[7] T. Hunter and A. Nosratinia, "Diversity through Coded Cooperation," IEEE Trans. Wireless Commun., vol. 5, no. 2, pp. 283-289, Feb. 2006

[8] C. Patel and G. Stüber, "Channel Estimation for Amplify and Forward Relay Based Cooperation Diversity Systems," IEEE Trans. Wireless Commun., vol. 6, no. 6, pp. 2348-2356, June 2007

[9] F. Gao, T. Cui and A. Nallanathan, "On Channel Estimation and Optimal Training Design for Amplify and Forward Relay Networks," IEEE Trans. on Commun., vol. 7, no. 5, pp. 1907-1916, May 2008

[10] B. Gedik and M. Uysal, "Two Channel Estimation Methods for Amplify-and-Forward Relay Networks," Canadian Conference on Electrical and Computer Engineering, 2010, pp. 615-618

[11] H. Guenach, M. Wymeersch and M. Moeneclaey, "On the Channel Parameter Estimation in a Space Time Bit-interleaved-coded Modulation System for Multipath DS-CDMA Uplink with Receive Diversity," IEEE Transactions on Vehicular Technology, vol. 54, no. 5, pp. 1747-1758, Sept. 2005

[12] H. Wymeersch, F. Simoens and M. Moeneclaey, "Code-aided Joint Channel Estimation and Frame Synchronization for MIMO Systems," IEEE 5th workshop on Signal Processing Advances in Wireless Communications, 2004, pp. 82-86

[13] D. Brennan, "Linear Diversity Combining Techniques," Proceedings of the IRE, vol. 47, no. 6, pp. 1075-1102, June 1959

[14] S. Lin and D. Costello Error Control Coding Pearson Education Inc., second edition, 2004

[15] A. J. Viterbi, 'Error Bounds for Convolutional Codes and an Asymptotically Optimum Decoding Algorithm," IEEE Transactions Information Theory, vol. 13, pp. 260-269, April 1967

[16] H. Wymeersch Iterative Receiver Design Cambridge University Press, 2007

[17] A. Dempster, N. Laird and D. B. Rubin, "Maximum Like-lihood From Incomplete Data Via the EM Algorithm," J. Royal Statistical Soc., vol. 39 , no. 1 , pp. $1-38,1977$

[18] H. L. Van Trees Detection, Estimation and Modulation Theory. New York: Wiley 1968

[19] A. D. Andrea, U. Mengali and R. Reggiannini , "The Modified CramerRao Bound and its Applications to Synchronization Problems," IEEE Trans. Commun., vol. 42, no. 234, pp. 1391-1399, Feb.-April 1994

[20] M. Moeneclaey, "On the True and the Modified Cramer-Rao Bounds for the Estimation of a Scalar Parameter in the Presence of Nuisance Parameters," IEEE Trans. Commun., vol. 46, no. 11, pp. 1536-1544, Nov. 1998

[21] N. Aerts, I. Avram and M. Moeneclaey, "The Cramer-Rao Bound for Channel Estimation in Block Fading Amplify-and-Forward Relaying Networks," IEEE 11th International Symposium on Spread Spectrum Techniques and Applications (ISITA), 2010, pp. 12-16 